\title{
Warfarin Use, Stroke, and Bleeding Risk among Pre-Existing Atrial Fibrillation US Veterans Transitioning to Dialysis
}

\author{
John Sy ${ }^{a}$ Cachet Wenziger ${ }^{a, b}$ Maria Marroquin ${ }^{a} \quad K^{2}$ Kamar Kalantar-Zadeh ${ }^{a, b}$ \\ Csaba Kovesdy ${ }^{c, d}$ Elani Streja ${ }^{a, b}$

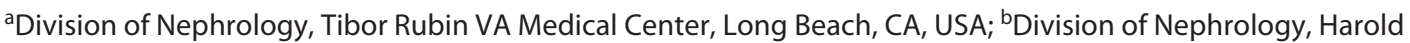 \\ Simmons Center for Kidney Disease Research and Epidemiology, Hypertension, and Kidney Disease, University \\ of California Irvine School of Medicine, Orange, CA, USA; 'Division of Nephrology, University of Tennessee Health \\ Science Center, Memphis, TN, USA; ${ }^{d}$ Nephrology Section, Memphis VA Medical Center, Memphis, TN, USA
}

\section{Keywords}

Anticoagulation - Warfarin - Dialysis · Atrial fibrillation .

Dialysis transition

\begin{abstract}
Introduction: Anticoagulation is commonly used for stroke prevention among patients with atrial fibrillation (AF); however, end-stage renal disease (ESRD) patients on hemodialysis are at higher risk of bleeding and stroke, even without anticoagulation. It is unclear if patients should be continued on anticoagulation at the time of transition to ESRD. In this study, we validated risk scores for stroke and bleeding in this population and assessed risk of stroke and bleeding among warfarin users compared to nonusers. Methods: We utilized a cohort of 28,620 pre-dialysis US veterans transitioning to hemodialysis between October 2007 and March 2015. Incident rates for the risks of stroke and bleeding were ascertained based upon $\mathrm{CHA}_{2} \mathrm{DS}_{2}$-VASc or HAS-BLED scores, respectively. A propensity score-based competing risk analysis was used to assess risk of stroke and bleeding. Findings: The mean age of our cohort was $77 \pm 9$ years, and the median $\mathrm{CHA}_{2} \mathrm{DS}_{2}$-VASc and HAS-BLED scores were $7(5,8)$ and $3(3$, $4)$, respectively. Increasing $\mathrm{CHA}_{2} \mathrm{DS}_{2}$-VASc and HAS-BLED
\end{abstract}

karger@karger.com

(c) 2022 S. Karger AG, Basel

www.karger.com/nef

Karger' scores were predictive of increasing stroke and bleeding rates, respectively. However, warfarin use did not appear to affect the risk of stroke and bleeding ( $p$-interaction $=0.84$ for stroke and 0.24 for bleeding). Warfarin use was associated with a higher risk of stroke (adjusted SHR 1.44, 95\% Cl: 1.231.69) and a higher risk of bleeding (adjusted SHR 1.38, 95\% $\mathrm{Cl}$ : 1.25-1.52) when accounting for the competing risk of death. Discussion: There was no difference in incidence rates of stroke or bleeding among warfarin users versus nonusers. Warfarin was associated with a higher risk of stroke and bleeding after considering mortality risk.

(c) 2022 S. Karger AG, Basel

\section{Introduction}

Atrial fibrillation (AF) is associated with a high risk of stroke, and among dialysis patients, the risk of stroke is even higher [1]. The mainstay of stroke prevention for AF has traditionally been the use of anticoagulation with warfarin and more recently with direct oral anticoagu-

John Sy and Cachet Wenziger contributed equally.
Correspondence to:

John Sy, john.sy@va.gov 
lants [2]. The clinical decision to initiate anticoagulation to prevent future stroke is typically weighed against the risk of bleeding, which is also more common among dialysis patients. Several risk scores have been developed to assist with determining this balance [3]. The most widely used scores, the $\mathrm{CHA}_{2} \mathrm{DS}_{2}$-VASc for stroke risk and HASBLED for bleeding risk, have provided clinicians with a tool to weigh these competing risks, but neither has been adequately validated among dialysis patients $[4,5]$. This decreases the reliability of these scores in this group of high-risk patients and may result in unnecessary or even harmful treatment with anticoagulation [6].

Furthermore, little is known about the risk of stroke and bleeding for patients who transition from nondialysis to dialysis. It is unclear how many of these patients continue prescriptions for anticoagulation and if these peritransition patients have similar stroke and bleeding risk compared to those who have been on dialysis for several years or have similar risk to those who have chronic kidney disease not on dialysis. Several recent studies have suggested that treating AF patients on dialysis with anticoagulation may in fact be associated with an increased risk of bleeding without a concomitant decrease in risk of stroke $[1,7,8]$. The debate on whether to anticoagulate dialysis patients with AF for primary stroke prophylaxis was also highlighted recently $[9,10]$.

In this study, we seek to determine the prevalence of warfarin use among those with AF both before and after transition to dialysis (i.e., dialysis initiation) and the prognostic ability of two widely used risk scores for assessing stroke and bleeding risk among AF patients, the $\mathrm{CHA}_{2} \mathrm{DS}_{2}$-VASc and HAS-BLED scores, respectively. We go on further to compare the risks of stroke and bleeding for patients transitioning to dialysis to determine if patients are likely to benefit from anticoagulation with warfarin.

\section{Methods}

\section{Study Population and Data Source}

We retrospectively examined data from the US Renal Data System (USRDS) Transition of Care in Chronic Kidney Disease (TCCKD) study [11-14], consisting of 102,477 adult US veterans who transitioned to hemodialysis between October 1, 2007, and March 31, 2015. The US Department of Veterans Affairs (VA) inpatient and outpatient records were examined using the International Classification of Diseases, Ninth Revision (ICD-9) codes for any $\mathrm{AF}$ event that occurred anytime during the study period. After excluding patients on direct oral anticoagulants anytime during the study period, our final cohort comprised 28,620 patients (online suppl. Fig. 1; see www.karger.com/doi/10.1159/000521494 for all online suppl. material). These pre-dialysis AF patients were examined for a warfarin prescription within the 6 months prior to the date of dialysis initiation through VA pharmacy data and the Centers for Medicare and Medicaid Services (CMS) Medicare Part $\mathrm{D}$ (prescription) data sources.

The pre-dialysis AF patients were then subdivided into four total groups based upon exposure to warfarin both before and after dialysis initiation (defined as those who had a prescription for warfarin within 90 days after the date of dialysis initiation, including at least a 5-day supply of warfarin and less than 90 days between refills). The study was reviewed by the Tibor Rubin and Memphis Veterans Affairs Medical Centers' Institutional Review Boards (IRB) and deemed exempt from the written consent requirement.

\section{Demographic and Clinical Data}

Baseline characteristics and demographics were obtained from a combination of three national data sources as follows: dialysis information was from USRDS and outcome and comorbid information was extracted from VA and Centers for Medicare and Medicaid (CMS) databases using ICD-9 and Current Procedural Terminology (CPT) codes.

\section{Statistical Analyses}

Atrial Fibrillation prior to and after Dialysis Transition

We first compared baseline characteristics of the four warfarin exposure groups. The groups were compared using ANOVA, Kruskal-Wallis test, or a $\chi^{2}$ test as appropriate for data type and distribution.

\section{Validation of Risk Scores}

We then proceeded to ascertain the incidence rate of stroke and bleeding risk based upon calculated risk scores $\left(\mathrm{CHA}_{2} \mathrm{DS}_{2}\right.$-VASc and HAS-BLED) for patients with AF at the time of dialysis initiation stratified by the four warfarin groups. A $\mathrm{CHA}_{2} \mathrm{DS}_{2}$-VASc score was ascertained using age, gender, and the presence of the following comorbid conditions: stroke, myocardial infarction, hypertension, ischemic heart disease, congestive heart failure, diabetes, and cerebrovascular disease. A modified HAS-BLED score was created using the presence of the following comorbid conditions: hypertension, renal or liver failure, ischemic stroke, bleeding tendency, coagulopathy, and drug or alcohol use. The labile international normalized ratio (INR) criteria were not used due to unavailability of INR values. Comorbidities used to calculate the risk scores were ascertained at the point of dialysis transition.

Relevant outcomes of ischemic stroke and bleeding events were ascertained by ICD-9 codes (online suppl. Table 1) that occurred after transition to dialysis. Follow-up time was calculated from the date of transition to dialysis until the first ischemic stroke or bleeding event, death, loss to follow-up, or the end of the study period (September 1, 2015), whichever occurred first. Data on mortality and outcomes were extracted from USRDS, VA, and CMS databases.

Stroke and Bleeding Risk - Propensity Score Analysis

To take into consideration the possibility of bias by indication, we proceeded to perform a propensity score (PS) analysis comparing a 1:1 matched cohort of warfarin versus nonwarfarin users after dialysis initiation. The post-dialysis transition warfarin-exposed groups were combined to define our warfarin user group while those patients who were not on warfarin after dialysis transi- 


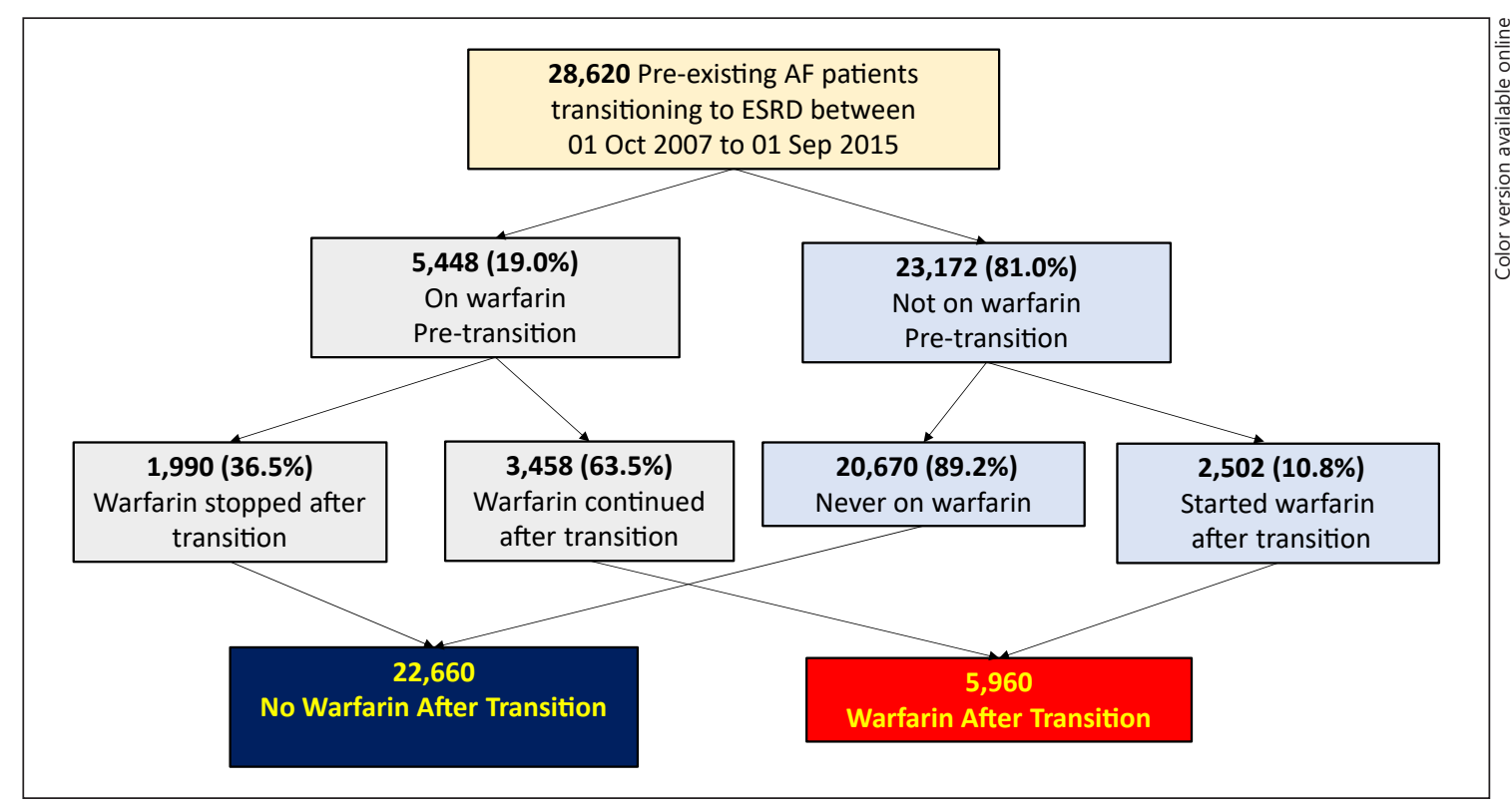

Fig. 1. Flowchart of warfarin use during the HD transition period among the 28,620 patients diagnosed with AF prior to HD transition.

tion were combined to define our nonwarfarin user group (Fig. 1). Variables used in the PS included age, gender, race, and comorbidities, as well as $\mathrm{CHA}_{2} \mathrm{DS}_{2}$-VASc and HAS-BLED scores, to match users of warfarin to nonusers after dialysis transition. Characteristics of patients in the PS matched cohort were compared with standardized mean differences, where a value 0.2 or greater is considered significant. We utilized a Cox model to ascertain the association between warfarin use after dialysis and both bleeding risk and stroke risk. Proportionality assumptions were checked in all models using Kaplan-Meier survival curves.

As a sensitivity analysis, we then proceeded to use a competing risk proportional hazards model (Fine-Gray) to consider the risk of death to ascertain both stroke and bleeding subdistribution hazard ratios (SHR) for users of warfarin versus nonwarfarin users after dialysis initiation. All analyses were performed with SAS, version 9.4 (SAS Institute Inc., Cary, NC, USA).

\section{Results}

Prevalence of Warfarin Use before and after Dialysis Transition

Of 28,620 patients who had AF prior to dialysis transition, 5,448 (19\%) were treated with warfarin within the 6 months prior to transition while 23,172 (81\%) were not treated with anticoagulation (Fig. 1). After dialysis initiation, 1,990 (37\%) individuals who were on warfarin prior to transition discontinued warfarin after dialysis transition while 3,458 (64\%) continued warfarin after dialysis transition.

\section{Demographics and Comorbidities}

The baseline characteristics at the time of dialysis transition are shown in Table 1 . Those with a warfarin prescription before transition only had the highest proportion of IHD and congestive heart failure (90\% and 93\%, respectively), and those with a warfarin prescription after transition had the highest proportion of diabetics (78\%) (Table 1).

\section{Stroke Rates by $\mathrm{CHA}_{2} \mathrm{DS}_{2}$-VASc Score by Warfarin Group}

Across all four warfarin status groups, an overall trend was seen for an increasing risk of stroke with increasing $\mathrm{CHA}_{2} \mathrm{DS}_{2}-\mathrm{VASc}$ scores. An interaction test showed that the effect of $\mathrm{CHA}_{2} \mathrm{DS}_{2}$-VASc scores on the risk of stroke did not significantly differ across warfarin status groups $(\mathrm{P}$ for interaction $=0.84)$ (Fig. 2 and online suppl. Table 2).

\section{Bleeding Rates by HAS-BLED Score by Warfarin Group}

An overall trend was seen for an increasing risk of bleeding with increasing HAS-BLED scores. An interaction test showed that the effect of HAS-BLED scores on the risk of bleeding did not significantly differ across warfarin status groups $(\mathrm{P}$ for interaction $=0.24)($ Fig. 3 and online suppl. Table 3). 
Table 1. Clinical characteristics of the cohort

\begin{tabular}{|c|c|c|c|c|c|c|}
\hline Female, $n(\%)$ & $1,238(4)$ & $927(5)$ & $68(3)$ & $109(4)$ & $134(4)$ & 0.0740 \\
\hline \multicolumn{7}{|l|}{ Race, $n(\%)$} \\
\hline White & $24,180(84)$ & $17,543(85)$ & $1,734(87)$ & $1,989(80)$ & $2,914(84)$ & $<0.0001$ \\
\hline Hispanic & $962(3)$ & $690(3)$ & $44(2)$ & $89(4)$ & $139(4)$ & 0.0045 \\
\hline Others & $695(2)$ & $514(2)$ & $30(2)$ & $69(3)$ & $82(2)$ & 0.0349 \\
\hline \multicolumn{7}{|l|}{ Comorbidities, $n$ (\%) } \\
\hline Cerebrovascular disease & $18,194(64)$ & $13,742(66)$ & $1,193(60)$ & $1,335(53)$ & $1,924(56)$ & $<0.0001$ \\
\hline Myocardial infraction & $14,802(52)$ & $11,074(54)$ & $1,045(53)$ & $1,137(45)$ & $1,546(45)$ & $<0.0001$ \\
\hline Peripheral vascular disease & $20,661(72)$ & $15,450(75)$ & $1,339(67)$ & $1,624(65)$ & $2,248(65)$ & $<0.0001$ \\
\hline Liver disease & $7,075(25)$ & $5,458(26)$ & $480(24)$ & $482(19)$ & $655(19)$ & $<0.0001$ \\
\hline Cancer & $12,295(43)$ & $9,412(46)$ & 779 (39) & $889(36)$ & $1,215(35)$ & $<0.0001$ \\
\hline Bleeding tendency & $10,810(38)$ & $7,932(38)$ & $791(40)$ & $822(33)$ & $1,265(37)$ & $<0.0001$ \\
\hline Coagulopathy & $5,749(20)$ & $3,756(18)$ & $628(32)$ & $422(17)$ & $943(27)$ & $<0.0001$ \\
\hline Drug use & $239(1)$ & $166(1)$ & $10(1)$ & $25(1)$ & $38(1)$ & 0.0845 \\
\hline Alcohol use & $1,274(4)$ & $916(4)$ & $91(5)$ & $136(5)$ & $131(4)$ & 0.0249 \\
\hline $\mathrm{CHA}_{2} \mathrm{DS}_{2}$-VASc score, median (IQR) & $7(5,8)$ & $7(6,8)$ & $7(5,8)$ & $6(5,7)$ & $6(5,7)$ & $<0.0001$ \\
\hline HAS-BLED score, median (IQR) & $3(3,4)$ & $3(3,4)$ & $4(3,4)$ & $3(2,4)$ & $3(3,4)$ & $<0.0001$ \\
\hline
\end{tabular}

Demographics and comorbidities for 28,620 TCCKD patients with stratification by warfarin prescription status before and after dialysis initiation (transition).

Fig. 2. From the 28,620 pre-HD AF patients, incidence rates of stroke events with stratification by warfarin prescription status before and after dialysis initiation (transition) by the $\mathrm{CHA}_{2} \mathrm{DS}_{2}$-VASc score.

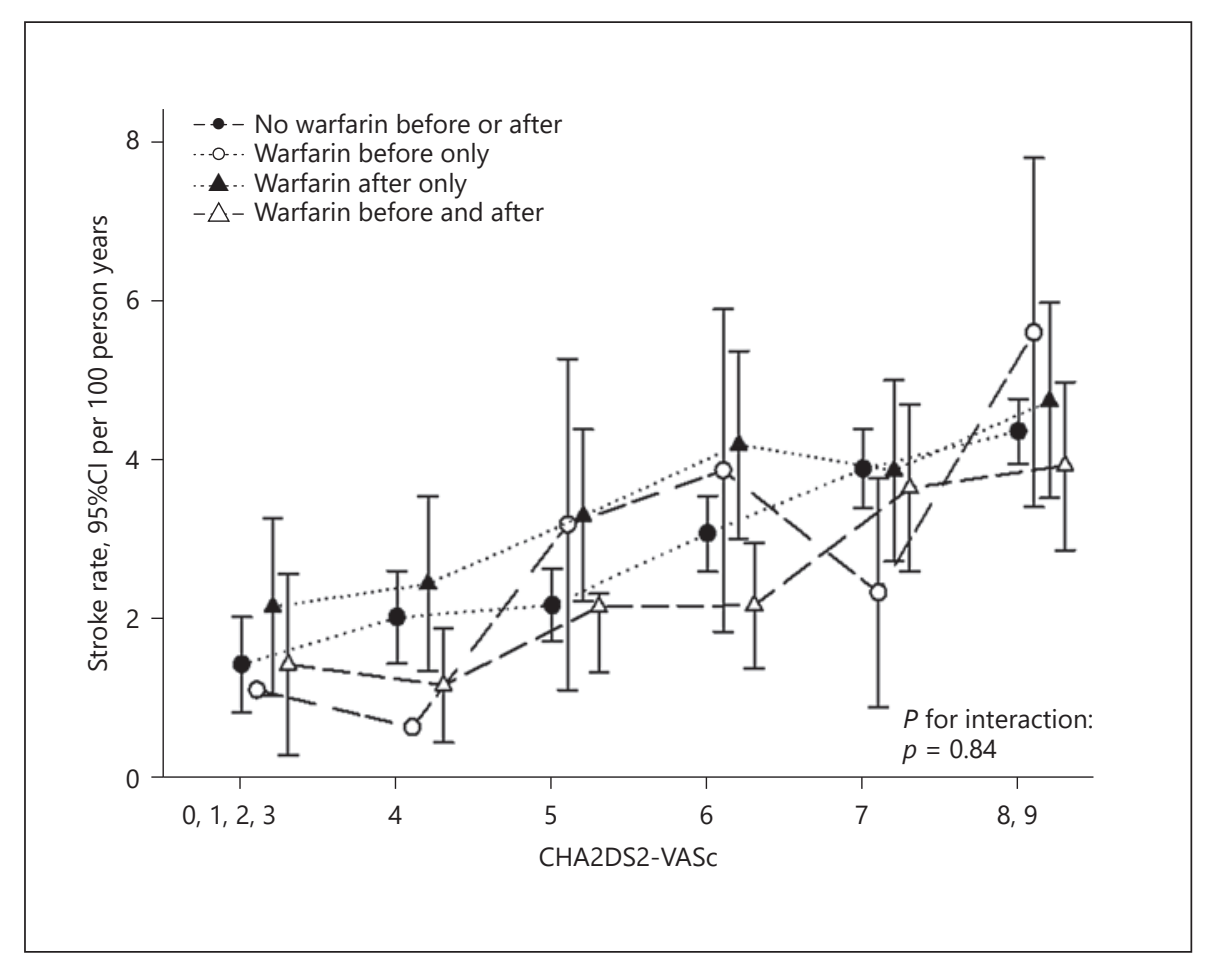


Table 2. Propensity score-matched cohort (1:1) characteristics

Fig. 3. From the 28,620 pre-HD AF patients, incidence rates of bleeding events with stratification by warfarin prescription status before and after dialysis initiation (transition) by the HAS-BLED score.

\begin{tabular}{|c|c|c|c|}
\hline Variable & $\begin{array}{l}\text { No warfarin after } \\
\text { transition } \\
(n=5,960)\end{array}$ & $\begin{array}{l}\text { Warfarin after } \\
\text { transition } \\
(n=5,960)\end{array}$ & $\begin{array}{l}\text { Standardized } \\
\text { difference }\end{array}$ \\
\hline Age, mean $\pm S D$, years & $74 \pm 9$ & $74 \pm 9$ & -0.01 \\
\hline Female, $n(\%)$ & $213(4)$ & $243(4)$ & 0.03 \\
\hline \multicolumn{4}{|l|}{ Race, $n(\%)$} \\
\hline White & $4,956(83)$ & $4,903(82)$ & -0.02 \\
\hline African American & $877(15)$ & $906(15)$ & 0.01 \\
\hline Hispanic & $181(3)$ & $228(4)$ & 0.04 \\
\hline Others & $127(2)$ & $151(3)$ & 0.03 \\
\hline \multicolumn{4}{|l|}{ Comorbidities, $n(\%)$} \\
\hline Cerebrovascular disease & $3,235(54)$ & $3,259(55)$ & 0.0081 \\
\hline Myocardial infraction & $2,747(46)$ & $2,683(45)$ & -0.0216 \\
\hline Peripheral vascular disease & $3,918(66)$ & $3,872(65)$ & -0.0162 \\
\hline Ischemic heart disease & $5,126(86)$ & $5,068(85)$ & -0.0277 \\
\hline Hypertension & $5,930(99)$ & $5,911(99)$ & -0.0398 \\
\hline Congestive heart failure & $5,247(88)$ & $5,171(87)$ & -0.0385 \\
\hline Diabetes & $4,696(79)$ & $4,648(78)$ & -0.0196 \\
\hline Liver disease & $1,133(19)$ & $1,137(19)$ & 0.0017 \\
\hline Cancer & $2,095(35)$ & $2,104(35)$ & 0.0032 \\
\hline $\mathrm{CHA}_{2} \mathrm{DS}_{2}$-VASc score, median (IQR) & $6(5,7)$ & $6(5,7)$ & 0.01 \\
\hline HAS-BLED score, median (IQR) & $3(3,4)$ & $3(3,4)$ & 0.01 \\
\hline
\end{tabular}

Clinical characteristics of 5,960 patients on warfarin versus 5,960 patients not on warfarin after dialysis transition.

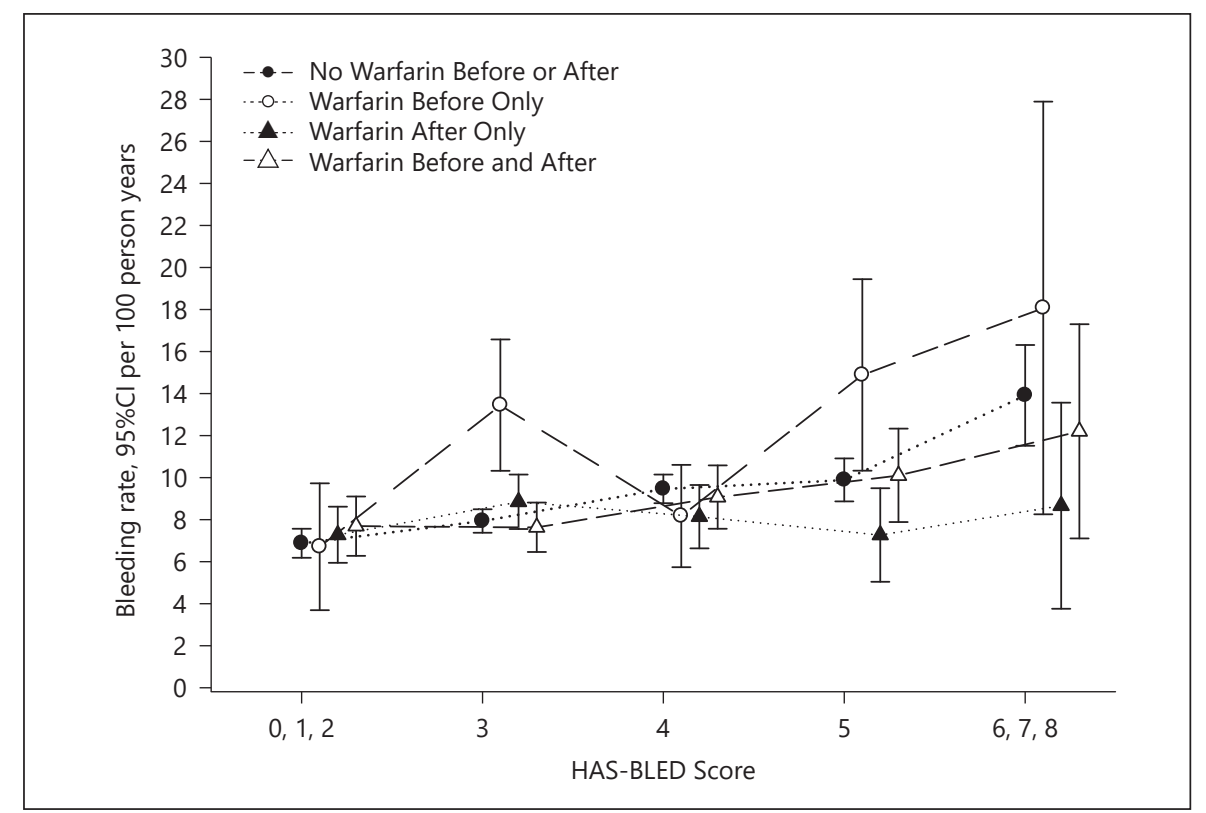

Propensity Score Analysis and Competing Risks

Proportional Hazards Regression for Stroke and

Bleeding Risk

For the PS analysis, the group of 3,458 patients who took warfarin both before and after transition was com- bined with the 2,502 who took warfarin after transition only to form a group consisting of 5,960 patients to serve as the exposure group when examining warfarin use after the HD transition period. The control group consisted of 22,660 patients from combining the 1,990 who took war- 


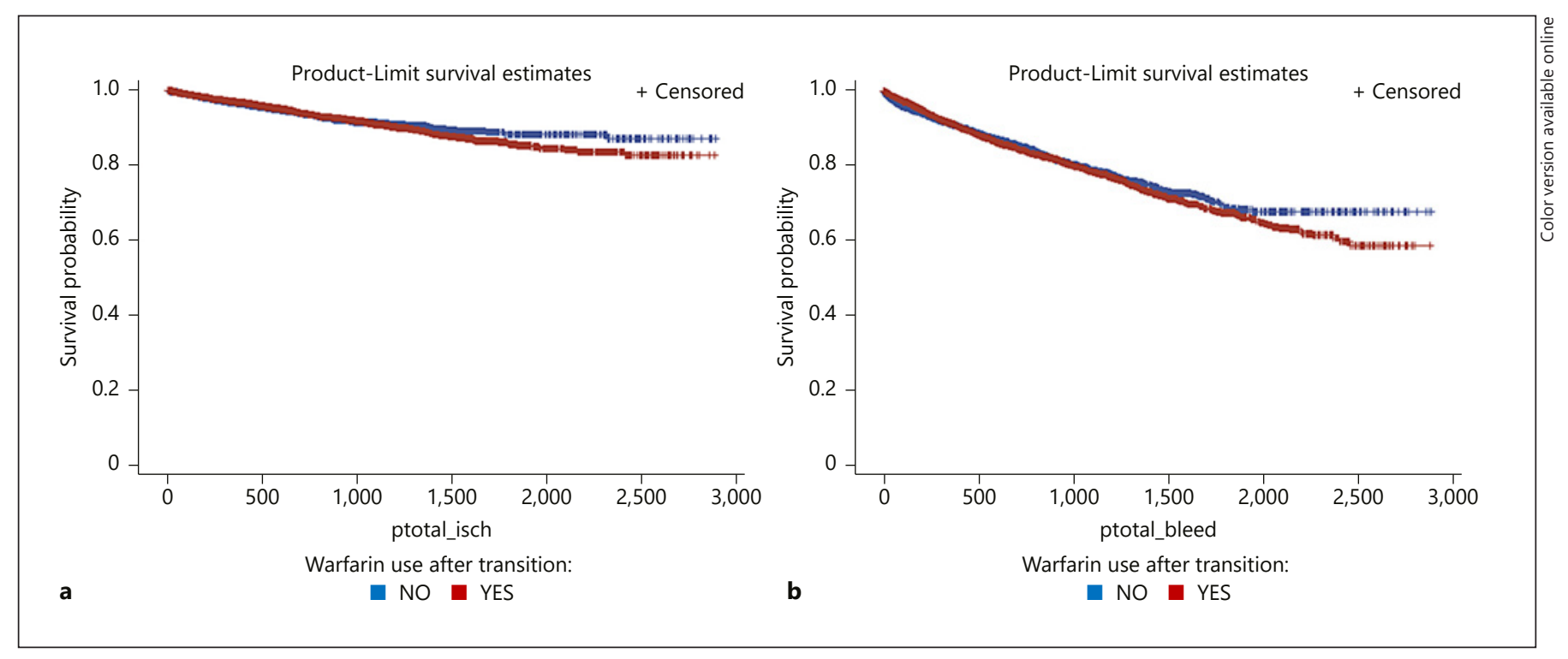

Fig. 4. Kaplan-Meier curves showing the probability of survival for stroke events (a) and bleeding events (b) stratified by warfarin users and nonusers after transition in the propensity score-matched cohort.

farin before transition only with the 20,670 who did not take warfarin either before or after transition.

The characteristics of the PS-matched cohort can be found in Table 2. The crude stroke rate was similar in patients with warfarin use after transition with a total of 388 (7\%) stroke events and a rate of 3.10 per 100 patient-years (95\% CI: 2.79-3.41) compared to those with no warfarin use after transition (259 events) and a stroke rate of 2.99 per 100 patient-years (95\% CI: 2.63-3.35) (Fig. 4a). The crude bleeding rate was also similar in patients with warfarin use after transition with a total of 973 (16\%) bleeding events and a rate of 8.27 per 100 patient-years $(95 \%$ CI: 7.76-8.80) compared to those with no warfarin use after transition (684 events) and a bleeding rate of 8.31 per 100 patient-years (95\% CI: 7.69-8.94) (Fig. 4b). Cox proportional hazards regression models in the PSmatched cohort showed no significant difference in risk of bleeding between those taking warfarin after transition compared to those not taking warfarin (HR 1.03, 95\% CI: $0.93-1.14, p=0.57)$. Those taking and not taking warfarin after transition also had similar risks of stroke (HR 1.04, $95 \%$ CI: $0.89-1.21, p=0.66$ ). The risk of all-cause mortality was $34 \%$ lower for those who took warfarin after transition (HR $0.66,95 \%$ CI: $0.64-0.69, p<0.01$ ) compared to those who were not taking warfarin.

Given the lower risk of death among warfarin users and the possibility of overestimating events with traditional time-to-event analysis, we used competing risks analyses (Fine-Gray) to consider the effect of mortality. In this analysis, warfarin users showed a $44 \%$ higher risk of stroke compared to nonusers after transition (SHR 1.44, 95\% CI: $1.23-1.69, p<0.01)$. When assessing bleeding risks, we found that warfarin users after transition had a 38\% higher risk of bleeding compared to nonusers after transition (SHR 1.38, 95\% CI: 1.25-1.52, $p<0.01$ ).

\section{Discussion}

In this study of US veterans, we found that approximately $29 \%$ of CKD stage 5 veterans who transitioned to hemodialysis had pre-existing AF. Of these, $19.0 \%$ were treated with warfarin within the 6 months prior to transition, and the majority (63.5\%) of these patients continued warfarin through dialysis. We also found that while higher $\mathrm{CHA}_{2} \mathrm{DS}_{2}$-VASc scores predicted higher risk of stroke, treatment with warfarin did not appear to have an effect at lowering stroke risk. Bleeding risk patterns were similar in that while higher HAS-BLED scores did predict higher bleeding risk, warfarin use was also not associated with a higher bleeding risk compared to nonwarfarin users. To mitigate issues due to confounding by indication as well as death as a competing risk, our PS competing risk analyses showed that warfarin users in fact had a $44 \%$ higher risk of stroke compared to nonusers and a $38 \%$ higher risk of bleeding compared to nonusers. 
Interestingly, $81.0 \%$ of patients with pre-existing $\mathrm{AF}$ prior to dialysis initiation were not on warfarin or any other anticoagulation prior to dialysis transition. Most of these patients had $\mathrm{CHA}_{2} \mathrm{DS}_{2}$-VASc scores above 2 (for female) and 3 (for male) under which the latest guidelines would recommend full anticoagulation [2]. While some comorbidities did show a significant trend between our four groups, there were no clear differences between those who were taking warfarin before dialysis transition and those who were not taking warfarin before dialysis transition. It is unclear if this was the result of a patient and provider discussion or a conscious decision to not initiate anticoagulation due to other factors. With regard to warfarin use after dialysis transition where guidelines are no longer as clear, we found that only $20.8 \%$ of patients with AF were on anticoagulation. Again, comparison of the groups who took warfarin after dialysis transition did not show any clear differences. These results are similar to a recent study showing that prevalence of warfarin use was approximately $22.0 \%$ in the ESKD population [15].

In the validation of the $\mathrm{CHA}_{2} \mathrm{DS}_{2}$-VASc score, our results suggested a lower risk of stroke compared to a study also attempting to validate the $\mathrm{CHA}_{2} \mathrm{DS}_{2}$-VASc score in a cohort of Taiwanese hemodialysis patients. Chao and colleagues [16] found that a $\mathrm{CHA}_{2} \mathrm{DS}_{2}$-VASc score of 0 was associated with an ischemic stroke incidence of 2.1 per $100 \mathrm{pt}$-years while the highest score of 9 was associated with an incidence rate of 13.1 per 100 pt-years (compared to our lowest stroke risk of 0.63 per 100 pt-years and highest stroke risk of 5.58 per 100 pt-years). Our average age was slightly higher at 77 years, we had a higher proportion of male patients, and we had a higher proportion of comorbid conditions in our cohort (specifically higher prevalence of heart disease, vascular disease, and diabetes) that may have resulted in differences in stroke risk. Our study continues to show that higher $\mathrm{CHA}_{2} \mathrm{DS}_{2}-$ VASc scores are associated with a higher risk of ischemic stroke, but our interaction analysis suggests that the stroke risk is not significantly different between our four groups. Similarly, we also show that increasing HASBLED scores are also associated with higher risk of major bleeding, but again, our interaction analysis suggests that the bleeding risk is also not significantly different between our four groups.

When using our PS analysis to assess risk of stroke and bleeding among those taking and not taking warfarin after dialysis transition, the results of this study add to a recently published study using the same cohort showing that warfarin use was associated with higher risk of stroke (HR 1.23, 95\% CI: 1.16-1.30) and bleeding events (HR 1.36,
95\% CI: 1.29-1.44), but a lower risk of death (HR 0.94, 95\% CI: 0.90-0.97) [14]. While the study sought to also assess the safety and efficacy of warfarin, it did not include prevalent AF patients, and our risk analysis varied by cohort entry date (time of warfarin initiation vs. time of dialysis transition for our study). In addition, given the lower risk of death among warfarin users, the study may have been prone to bias by indication. In this study, we improved the risk estimates of stroke and bleeding risk by utilizing a competing risk analysis in a PS which showed that warfarin use was actually associated with an increase in both stroke and bleeding risk by $44 \%$ and $38 \%$, respectively.

Previous studies have been inconclusive as to whether warfarin is beneficial in reducing stroke risk in dialysis patients, but studies from late-stage CKD appear to support the use of warfarin to reduce stroke $[9,17]$. Whether or not patients at the point of dialysis transition have risks similar to established dialysis patients or late-stage CKD patients was unclear, and our study suggests that these patients have risks like their established dialysis peers in that the risk of stroke is not significantly improved with the use of warfarin for anticoagulation. Furthermore, while previous studies have potentially suggested no differences in stroke risk with use of warfarin [15, 17], our PS competing risk analysis suggests that warfarin users may have a higher risk of stroke compared to nonwarfarin users.

With regard to bleeding risks, it also appears that patients at the point of dialysis transition have risks more like established hemodialysis patients compared to latestage CKD patients. While one meta-analysis of dialysis patients did not show an increased risk of major bleeding (HR 1.20, 95\% CI: 0.99-1.47) [15], another meta-analysis of a similar cohort showed that warfarin was associated with an increased risk of major bleeding (HR 1.30, 95\% CI: 1.08-1.56) [17]. Our competing risk PS analyses taking death into consideration did show that warfarin users have a higher risk of bleeding events compared to nonusers which is in line with the latter meta-analysis.

While our large patient population strengthens our study, we do acknowledge some limitations. There are many unmeasured confounders in the association between warfarin use and outcomes including potential confounding by indication. We attempted to address these issues using a PS competing risk analysis to mitigate confounding by indication bias as well as any potential biases that death may have had on outcomes. In addition, the use of ICD-9 codes may not accurately capture diagnoses or outcomes, though our prevalence of AF among dialysis patients is in line with previously reported studies suggesting our capture method is reasonable. We also ac- 
knowledge the lack of information to calculate the full HAS-BLED score, but our modified HAS-BLED score remains a robust assessment of bleeding risk in our dialysis patients and can help inform future bleeding risk of patients on and off warfarin. Further studies should be done to assess stroke and bleeding risks among late-stage CKD patients, especially if it is unclear why many of these patients were not on anticoagulation prior to dialysis transition despite having a guideline-based indication.

In conclusion, our study provides evidence that warfarin users do not appear to be associated with a lower risk of stroke nor a higher risk of bleeding compared to those not taking warfarin. Furthermore, warfarin appears to be associated with a significantly higher bleeding and stroke risk compared to nonusers in our PS competing risk analysis suggesting that warfarin use should be re-evaluated at the time of dialysis transition. As there is a higher possibility of stroke and bleeding outcomes among warfarin users compared to nonusers, we recommend that warfarin not be used for primary stroke prophylaxis among hemodialysis patients with AF. However, newer anticoagulation agents are now available with evidence suggesting that these medications may be a safer alternative compared to warfarin $[18,19]$. Further studies are needed to determine if these pre-existing AF patients transitioned to dialysis can benefit from starting these newer agents.

\section{Acknowledgment}

Preliminary findings were presented as an oral abstract at the American Society of Nephrology 2020 Kidney Week Reimagined.

\section{Statement of Ethics}

The study was reviewed by the Tibor Rubin and Memphis Veterans Affairs Medical Centers' Institutional Review Boards (IRB) and deemed exempt from the written consent requirement.

\section{Conflict of Interest Statement}

The authors report no conflicts of interest relevant to the manuscript.

\section{Funding Sources}

The work in this article has been performed with the support of the National Institute of Diabetes and Digestive and Kidney Diseases (NIDDK) of the National Institutes of Health (NIH) research grant U01-DK102163 to C.P.K. and K.K.Z., and by resources from the US Department of Veterans Affairs. Support for Veterans Health Administration/CMS data are provided by the Department of Veterans Affairs, Veterans Health Administration, Office of Research and Development, Health Services Research and Development, Veterans Affairs Information Resource Center (project numbers SDR 02-237 and 98-004). K.K.Z. has been supported by the NIH/NIDDK midcareer award K24-DK091419. E.S. is supported by a career development award from the Office of Research and Development of the Department of Veterans Affairs (IK2CX001266-01). J.S., C.P.K., K.K.Z., and E.S. are employees of the VA. The interpretation and reporting of these data are the responsibility of the authors and in no way should be seen as official policy or interpretation of the Department of Veterans Affairs or the US government.

\section{Author Contributions}

J.S., C.W., C.K., K.K.Z., and E.S. contributed to writing the manuscript. C.W., M.M., and E.S. contributed to data acquisition and processing. J.S., C.W., M.M., and E.S. contributed to data analysis. The authors had full access to the data in the study and take responsibility for the integrity of the data and the accuracy of the data analysis.

\section{Data Availability Statement}

Data used in this study were made available by the US Department of Veterans Affairs and can be requested through the VA Office of Research and Development (ORD).

\section{References}

1 Kuno T, Takagi H, Ando T, Sugiyama T, Miyashita S, Valentin N, et al. Oral anticoagulation for patients with atrial fibrillation on long-term dialysis. J Am Coll Cardiol. 2020 Jan;75(3):273-85.

2 January CT, Wann LS, Calkins H, Chen LY, Cigarroa JE, Cleveland JC, et al. 2019 AHA/ ACC/HRS focused update of the 2014 AHA/ ACC/HRS guideline for the management of patients with atrial fibrillation: a report of the American College of Cardiology/American Heart Association Task Force on clinical practice guidelines and the heart rhythm society. Circulation. 2019 Jul;140(2):e125-51.

3 van den Ham HA, Klungel OH, Singer DE, Leufkens HG, van Staa TP. Comparative performance of ATRIA, CHADS2, and CHA2DS2-VASc risk scores predicting stroke in patients with atrial fibrillation: results from a national primary care database. J Am Coll Cardiol. 2015 Oct;66(17):1851-9.

4 Lip GY, Nieuwlaat R, Pisters R, Lane DA, Crijns HJ. Refining clinical risk stratification for predicting stroke and thromboembolism in atrial fibrillation using a novel risk factorbased approach: the euro heart survey on atrial fibrillation. Chest. $2010 \mathrm{Feb} ; 137(2): 263-72$.

5 Pisters R, Lane DA, Nieuwlaat R, de Vos CB, Crijns HJGM, Lip GYH. A novel user-friendly score (HAS-BLED) to assess 1-year risk of major bleeding in patients with atrial fibrillation. Chest. 2010 Nov; 138(5):1093-100.

6 Molnar AO, Sood MM. Predicting in a predicament: stroke and hemorrhage risk prediction in dialysis patients with atrial fibrillation. Semin Dial. 2018 Jan;31(1):37-47. 
7 Ha JT, Neuen BL, Cheng LP, Jun M, Toyama T, Gallagher MP, et al. Benefits and harms of oral anticoagulant therapy in chronic kidney disease: a systematic review and meta-analysis. Ann Intern Med. 2019 Aug;171(3):181-9.

8 Tsai C, Marcus LQ, Patel P, Battistella M. Warfarin use in hemodialysis patients with atrial fibrillation: a systematic review of stroke and bleeding outcomes. Can J Kidney Health Dis. 2017 Jan;4:2054358117735532.

9 Keskar V, Sood MM. Use of oral anticoagulation in the management of atrial fibrillation in patients with ESRD: con. Clin J Am Soc Nephrol. 2016 Nov;11(11):2085-92.

10 McCullough PA, Ball T, Cox KM, Assar MD Use of oral anticoagulation in the management of atrial fibrillation in patients with ESRD: pro. Clin J Am Soc Nephrol. 2016 Nov; 11(11):2079-84.

11 Streja E, Gosmanova EO, Molnar MZ, Soohoo M, Moradi H, Potukuchi PK, et al. Association of continuation of statin therapy initiated before transition to chronic dialysis therapy with mortality after dialysis initiation. JAMA Netw Open. 2018 Oct;1(6):e182311.
12 Molnar MZ, Gosmanova EO, Sumida K, Potukuchi PK, Lu JL, Jing J, et al. Predialysis cardiovascular disease medication adherence and mortality after transition to dialysis. Am J Kidney Dis. 2016 Oct;68(4):609-18.

13 United States Renal Data System. Chapter 8: transition of care in chronic kidney disease. Bethesda, MD: National Institute of Diabetes and Digestive and Kidney Diseases, National Institutes of Health; 2017. p. 46.

14 Agarwal MA, Potukuchi PK, Sumida K, Naseer A, Molnar MZ, George LK, et al. Clinical outcomes of warfarin initiation in advanced chronic kidney disease patients with incident atrial fibrillation. JACC Clin Electrophysiol. 2020 Dec;6(13):1658-68.

15 Randhawa MS, Vishwanath R, Rai MP, Wang L, Randhawa AK, Abela G, et al. Association between use of warfarin for atrial fibrillation and outcomes among patients with end-stage renal disease: a systematic review and metaanalysis. JAMA Netw Open. 2020 Apr;3(4): e202175.
16 Chao TF, Liu CJ, Wang KL, Lin YJ, Chang SL, Lo LW, et al. Incidence and prediction of ischemic stroke among atrial fibrillation patients with end-stage renal disease requiring dialysis. Heart Rhythm. 2014 Oct;11(10): 1752-9.

17 Dahal K, Kunwar S, Rijal J, Schulman P, Lee J. Stroke, major bleeding, and mortality outcomes in warfarin users with atrial fibrillation and chronic kidney disease: a meta-analysis of observational studies. Chest. 2016 Apr; 149(4):951-9.

18 Sy J, Hsiung JT, Edgett D, Kalantar-Zadeh K, Streja E, Lau WL. Cardiovascular and bleeding outcomes with anticoagulants across kidney disease stages: analysis of a national US cohort. Am J Nephrol. 2021 Mar;52(3):199_ 208

19 De Vriese AS, Caluwé R, Van Der Meersch H, De Boeck K, De Bacquer D. Safety and efficacy of vitamin K antagonists versus rivaroxaban in hemodialysis patients with atrial fibrillation: a multicenter randomized controlled trial. J Am Soc Nephrol. 2021 Jun;32(6):147483. ASN.2020111566. 